\title{
Adaptive Learning Algorithm for SVM Applied to Feature Tracking
}

\author{
Ashutosh Garg, Ira Cohen, Thomas S. Huang \\ University of Illinois at Urbana Champaign \\ Email: \{ashutosh,iracohen,huang\}@ifp.uiuc.edu
}

\begin{abstract}
The framework of Support Vector Machines is becoming extremely popular in the field of statistical pattern classification. Kalman filters have been used for long for doing tracking. In this paper we have investigated a technique which couples Kalman filter closely with the SVM. The problem of object tracking can be seen as a pattern recognition problem in which we are looking for a pattern (object to be tracked) in an image. However, because of the dynamics, this pattern might experience some changes over time. In order to keep track of the position of the pattern and to make out the desired pattern from the background (which may contain similar patterns), we must have some strong continuous time model. In this paper we have proposed an algorithm which combines the Markov property of the Kalman filter with the strong classification capability of SVM. The whole system has been tested on real life problems and we have discovered that with this framework we can track a particular object even in a frame which contains identical objects. The results are compared to that of obtained by color blob tracking which show the strength of the approach. This will be extremely useful in the environments in which we want to track a particular person when more then one person is present.
\end{abstract}

\section{Introduction}

The purpose of this paper is to present an algorithm that provides an adaptive learning environment for the Support Vector Machines so that they can be used for robust feature tracking in video sequences. The SV algorithm is a nonlinear generalization of the Generalized Portrait algorithm developed in Russia in the sixties [10]. The Support Vector Machine is a novel type of learning machine based on statistical learning theory.

The standard training algorithms for SVMs are developed with the problem of pattern recognition in mind. As such, a database is used to train the SVMs and once trained, a hyperplane seperating the two classes is obtained. This has an inherent assumption that we don't expect any changes in the classes with time. Thus this cannot be used in its original form in the applications like tracking. When we are tracking a human body, the shape is always subject to changes, the position is bound to change, and as the person moves in a 3-D environment, the lighting changes. All these need to be taken care of when we are tracking. Some of the recent work on tracking $[1,7,8,13,14]$ provides answer to these by combining shape, color and motion to some extent. In present form, our algorithm provides a solution to some of these problems. The solution lies in using the color, shape and motion together as feature vectors and making the hyperplane and the support vectors follow the changes in these features.

The motivation for our algorithm comes form LMS algorithm which is used extensively in signal processing and where the system keeps track of the changes in the data by constantly updating the filter coefficients. In our algorithm we use the color and the object position as the feature vectors. The SVM is trained for these feature vectors. A Kalman filter [12] is used to keep track of the dynamics of the object. The support vectors are updated using the Kalman filter and then classification is done using the new support vectors (the hyperplane obtained from them). Once the classification is done, a new set of support vectors is obtained and the process is repeated.

\section{Support Vector Machines}

This section briefly describes the SVM (support vector machine) and gives the motivation behind using them. The detailed analysis of SVMs can be found in many papers such as [3]. It has been proved [11] that while doing binary classification, there exists an upper bound (Eq 1) on the expected risk which is independent of the probabilistic distribution of the data and the classes 


$$
\begin{gathered}
R(\alpha) \leq R_{e m p}(\alpha)+\sqrt{\left(\frac{h(\log (2 l / h)+1)-\log (\eta / 4)}{l}\right)} \\
R(\alpha)=\int \frac{1}{2}|d-f(\mathbf{x}, \alpha)| d P(\mathbf{x}, d) \\
R_{e m p}(\alpha)=\frac{1}{2 l} \sum_{i=1}^{l}\left|d_{i}-f\left(\mathbf{x}_{i}, \alpha\right)\right|
\end{gathered}
$$

Where $R_{e m p}$ is the empirical risk as given by $\mathrm{Eq}$ 2 and $R(\alpha)$ gives the actual risk. 'h' is known as the VC-dimension. VC dimension for a set of functions $f(\alpha)$ is defined as the maximum no. of training points that can be shattered by $f(\alpha) . P(\mathbf{x}, d)$ is the unknown probability distribution from which data is drawn. $f(\mathbf{x}, \alpha)$ is a mapping from $\mathbf{x} \rightarrow d$.

We start with a simple case of two linearly separable classes. Let us assume that there is a data set $D=$ $\left(\mathbf{x}_{i}, d_{i}\right)^{l}{ }_{i=1}$ of labeled examples, where $d_{i} \in\{-1,1\}$ and $x_{i} \in R^{n}$. We want to obtain the linear classifier that can separate the data based on the classification $d_{i}$ and at the same time minimize the empirical risk. This is known to be the hyperplane which provides the maximum margin. This can be formulated as follows:

$$
\begin{aligned}
& \mathbf{x}_{i} \cdot \mathbf{w}+b \geq+1 \text { for } d_{i}=+1 \\
& \mathbf{x}_{i} \cdot \mathbf{w}+b \leq-1 \text { for } d_{i}=-1
\end{aligned}
$$

where $\mathbf{w}$ is the normal to the hyperplane, $|b| /|| \mathbf{w}||$ is the perpendicular distance from the hyperplane to the origin, and $\|\mathbf{w}\|$ is the Euclidean norm of $\mathbf{w}$. The solution to this problem is expected to have the form as shown in Fig 1 . Let $d_{+}, d_{-}$be the shortest distance of points from the hyperplane belonging to different classes, then $d_{+}+d_{-}$defines the "margin" for the hyperplane. It can be seen that the solution of these two equations gives the margin as $2 /\|\mathbf{w}\|$. Thus the problem reduces to finding the hyperplane with maximum margin by minimizing $\|\mathbf{w}\|^{2}$. The points for which the equality holds in these two equations are known as support vectors and it can be seen that it is the movement of these points which controls the hyperplane.

The Lagrangian formulation of this problem goes as

$$
L_{p} \equiv\|\mathbf{w}\|^{2}-\sum_{i=1}^{l} \alpha_{i} d_{i}\left(\mathbf{x}_{i} \cdot \mathbf{w}+b\right)+\sum_{i=1}^{l} \alpha_{i}
$$

Where $\alpha_{i}, \mathrm{i}=1, \ldots, \mathrm{l}$ are the Lagrange multipliers, one for each inequality constraint in Eq 4,5. This $L_{p}$ is then minimized with respect to $\mathbf{w}, b$ and simultaneously requires that the derivatives of $L_{p}$ with respect to all $\alpha_{i}$ vanish. For the dual of this problem it turns out that we want to maximize,

$$
L_{d}=\sum_{i=1} \alpha_{i}-\frac{1}{2} \sum_{i, j} \alpha_{i} \alpha_{j} d_{i} d_{j} \mathbf{x}_{i} \cdot \mathbf{x}_{j}
$$

under the constraints (obtained from the gradient of $L_{P}$ )

$$
\begin{gathered}
\mathbf{w}=\sum_{i=1} \alpha_{i} d_{i} \mathbf{x}_{i} \\
\sum_{i=1} \alpha_{i} d_{i}=0
\end{gathered}
$$

In the solution those points for which $\alpha_{i}>0$ are called "support vectors". All the other training points have $\alpha_{i}=0$. Support vectors are the most critical elements of the training set. They lie closest to the decision boundary and if a new hyperplane is sought, using just these set of points the same result would be obtained.

Now, how do we generalize this to the case when we can't obtain a linear hyperplane i.e. in case when the decision function is not a linear function of the data. In the training algorithm above we notice that the data appears just in the form of dot products, $\mathbf{x}_{i} \cdot \mathbf{x}_{j}$. Now, if we first map the data to some higher dimensional space $H$, using mapping $\Phi$ :

$$
\Phi: \mathbf{R}^{\mathbf{d}} \rightarrow H
$$

Then in this case training the SVM would just depend on the dot product of the vectors in $H$, which implies computing $\Phi\left(\mathbf{x}_{i}\right) \cdot \Phi\left(\mathbf{x}_{j}\right)$. This may appear to be computationally expensive, but if there exists a "kernel function" $\mathrm{K}$, such that $K\left(\mathbf{x}_{i}, \mathbf{x}_{j}\right)=\Phi\left(\mathbf{x}_{i}\right) \cdot \Phi\left(\mathbf{x}_{j}\right)$, we would need only to use $\mathrm{K}$ in our training algorithm without ever explicitly knowing what $\Phi$ is. For some Kernel function to be a dot product in some space, it should satisfy the Mercer's condition [11]. Some of the kernel functions known to satisfy this condition are Polynomial classifier, Gaussian Radial Basis Function Classifier and Sigmoidal neural network.

\section{Updating Support Vectors}

In the previous section we have seen that classification problem can be reduced to the following

$$
f(\mathbf{x})=\operatorname{sgn}((\mathbf{w} \cdot \mathbf{x})+b)
$$




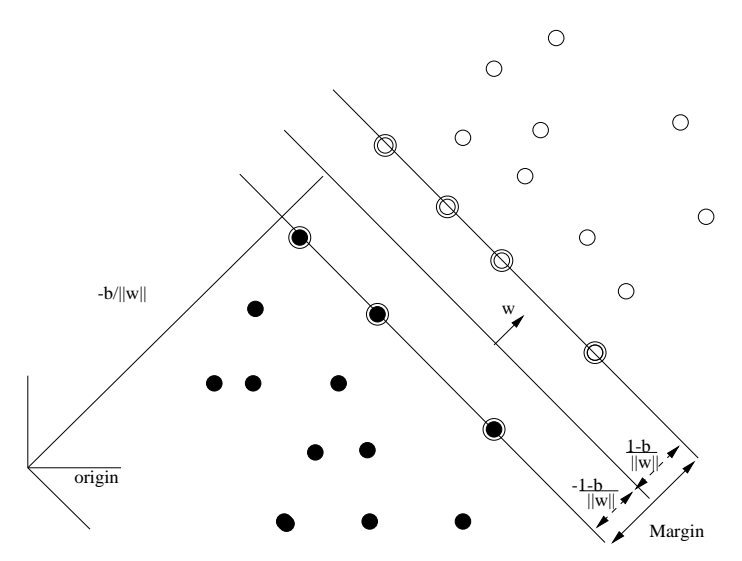

Figure 1: Linear hyperplane for linearly seperable case. The support vectors are circled.

Where $\mathbf{w}$ is normal to the hyperplane and $|b| /|| \mathbf{w}||$ is the distance of the hyperplane from the origin. This equation is the direct result of the Eq 4,5 given in the previous section. Suppose some vector $\mathbf{x}$ is translated by $\mathbf{v}$ to give $\mathbf{x}^{\prime}$. Then this equation changes to

$$
f\left(\mathbf{x}^{\prime}\right)=\operatorname{sgn}((\mathbf{w} \cdot(\mathbf{x}+\mathbf{v}))+b) \equiv \operatorname{sgn}\left((\mathbf{w} \cdot \mathbf{x})+b^{\prime}\right)
$$

where $b^{\prime}=b+\mathbf{w} \cdot \mathbf{v}$. In a particular case if only the vectors corresponding to one class (say + ve class) have moved then

$$
\begin{gathered}
d_{+}=\frac{1-b-\mathbf{w} \cdot \mathbf{v}}{\|\mathbf{w}\|} \\
d_{-}=\frac{-1-b}{\|\mathbf{w}\|}
\end{gathered}
$$

In this case, to maintain the maximum margin, the hyperplane must move keeping the same normal $\mathbf{w}$, by the distance $\frac{\mathbf{w} \cdot \mathbf{v}}{2 *\|\mathbf{w}\|}$. This is demonstrated for the 2-dimensional case in Fig 1,2.

In our case we know that the pixels corresponding to the object have moved. Therefore we need to move the hyperplane so as to maintain the maximum margin. However, we don't know the exact mapping to the higher dimensional space in which the hyperplane lies (Classification in that case is done using a kernel function). To achieve this we actually move the support vectors and then the hyperplane is obtained again. Since the no. of support vectors is very low, the computation cost is negligible.

To test this concept, we made use of the existing SVM tools. We started by creating a $(8 \mathrm{x} 6)$ pixel image in which a set of four pixel is marked as the object of interest (which is to be tracked).

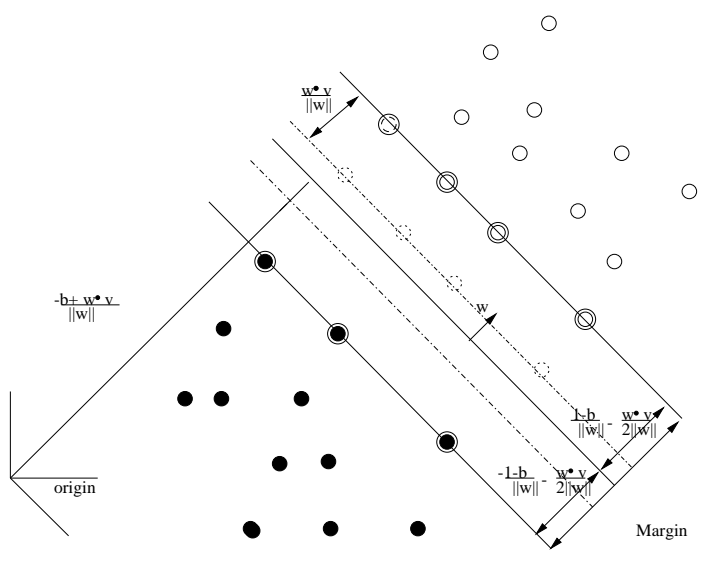

Figure 2: Shift in linear hyperplane as the points belonging to the positive classification drifts. Dotted line shows the hyperplane before the shift. Support Vectors are circled.

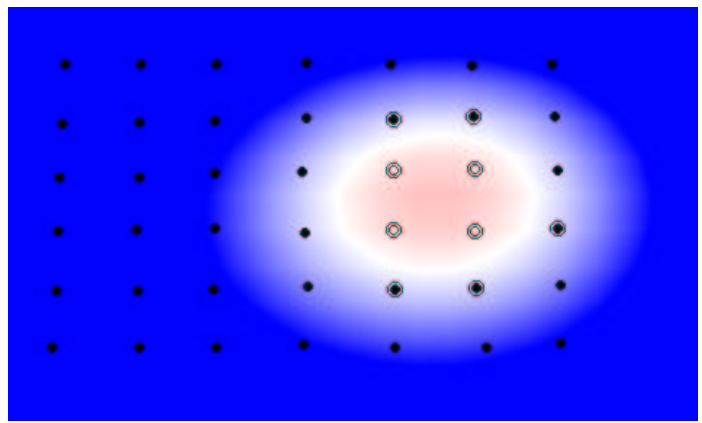

Figure 3: Original Image in which the four white pixels are the one that belongs to the object of interest. The pixels encircled are the support vectors.

Now we gave a predetermined motion of three unit to the pixels of the object and the what we observe is that the support vectors obtained in the new case have actually experienced the same motion. Fig 3 is the original image and Fig 4 is the image in which object has drifted by three units.

Thus it supports our basic idea that we can keep track of the blob by keeping track of the support vectors and at each time we just need to estimate the position of few support vectors instead of all the object points.

\section{Adaptive Learning Algorithm}

Once we know how SVM can be trained, how do we use them for the purpose of tracking? We start by 


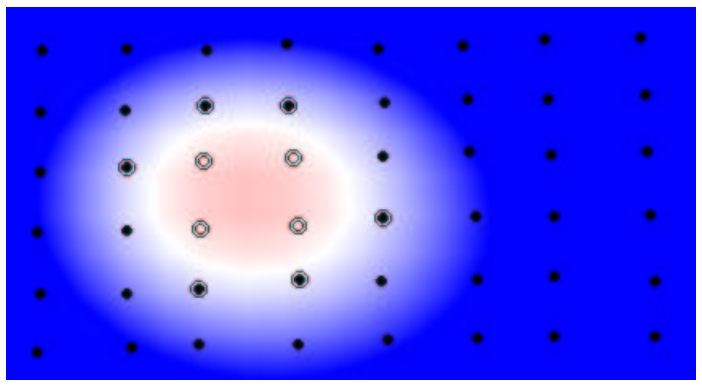

Figure 4: Hyperplane has shifted as the object has drifted to the left by three units. Support Vectors are circled.

analyzing the characteristics of the problem. For example we are interested in tracking the human arm robustly, which can be represented as a blob. The main characteristics that we are going to exploit are the color and shape of the blob. We know that skin has a color which can be used to distinguish it from the background. However, some objects in the image may have the same color. A standard approach to achieve this [7],[1] is to threshold the image using skin color as foreground and everything else as background. But this technique doesn't give good segmentation. It has been found that either position or shape is not a strong enough cue to segment and track an object. Combination of these two cues is one approach towards providing a robust tracking system.

The Algorithm that we are proposing can be summarized in the following steps.

\subsection{Step 1: Initialization and Training}

The first step in the process is to train the SVM. We start with defining a vector

$$
\mathbf{x}_{i}=\left\{x_{i}, y_{i}, r_{i}, g_{i}, b_{i}\right\}, \mathbf{x} \in \mathbf{R}^{5}
$$

where $x_{i}, y_{i}$ are the coordinates of the pixel and $r_{i}, g_{i}, b_{i}$ gives the color of the pixel.

Vector $d_{i}$ is taken to be the decision statistics for this data. For training the SVM we need the classified data and for this we need to have some sort of initialization algorithm which can separate the data into the set of pixels belonging to the object of interest and the ones belonging to the background. Right now this is achieved manually. This data is used to train the SVM using an appropriate kernel function( The one which can best capture the properties of the data set. This depends on the shape and other properties of the data). For capturing the dynamics of the object, we assume a constant velocity model Kalman filter. Let $\mathbf{p}_{\mathbf{k}}$ denote the state of the filter (which corresponds to the blobs position and velocity) and $\mathbf{z}_{\mathbf{k}}$ is the observation. Then we can initialize our filter as

$$
\begin{gathered}
\mathbf{p}_{k+1}=A_{k} \mathbf{p}_{k}+w_{k} \\
\mathbf{z}_{k}=\mathbf{p}_{k}+v_{k}
\end{gathered}
$$

with

$$
p(w) \sim N(0, Q), \quad p(v) \sim N(0, R)
$$

Where $A_{k}$ is a constant velocity model. $v_{k}, w_{k}$ represents the state noise and measurement noise respectively, which are assumed to be gaussian. This model is used to keep track of the position of the blob, predict the new position in the next frame and also to update the support vectors for classification.

\subsection{Step 2: Estimation of Velocity}

The blob is obtained from the classified data and the center of the blob gives its position. This position vector is the input to the Kalman Filter. Given the state of the system at time $\mathrm{k}$, we can predict the next state as

$$
\begin{gathered}
\hat{\mathbf{p}}_{k+1}=A_{k} \hat{\mathbf{p}}_{k} \\
P_{k+1}^{-}=A_{k} P_{k} A_{k}^{T}+Q_{k}
\end{gathered}
$$

Where Eq 20 is the update equation for the error covariance matrix. The predicted state gives the estimated position of the blob in the next frame.

\subsection{Step 3: Update the Support Vectors}

The Support vectors are updated using the predicted position of the blob given by Kalman filter. The difference between the predicted position of the blob and the measured position of the blob in the previous frame gives the estimated motion that is used to update the support vectors. We assume that the positive support vectors are going to experience this motion and thus are updated accordingly. As was mentioned in the previous section, the support vectors are the only points which determine the hyperplane (e.g. in case of non linear hyperplane, this turns out to be solving the kernel function using new support vectors). Using these SVs we obtain a new hyperplane which is used for classification in the next frame.

\subsection{Step 4: Object Classification}

Once the new image is obtained, pixels are classified as the ones belonging to the object of interest and 
the ones belonging to the background by solving the following equation.

$$
f(\mathbf{x})=\operatorname{sgn}\left(\sum_{i=1}^{l} d_{i} \alpha_{i} \cdot\left(K\left(\mathbf{x} \cdot \mathbf{s}_{\mathbf{i}}\right)\right)+b\right)
$$

where $\mathbf{s}_{i}$ are the support vectors obtained in the previous step and $K$ is the kernel function that is being used. $\alpha_{i}$ are the lagrangian multipliers that are obtained as a result of training the SVM. The new object is going to be close (in the $R^{5}$ space) to the one that was predicted. However, it may not be exactly the same as there might have been slight variations in the lighting conditions and due to errors in the dynamic estimation model.

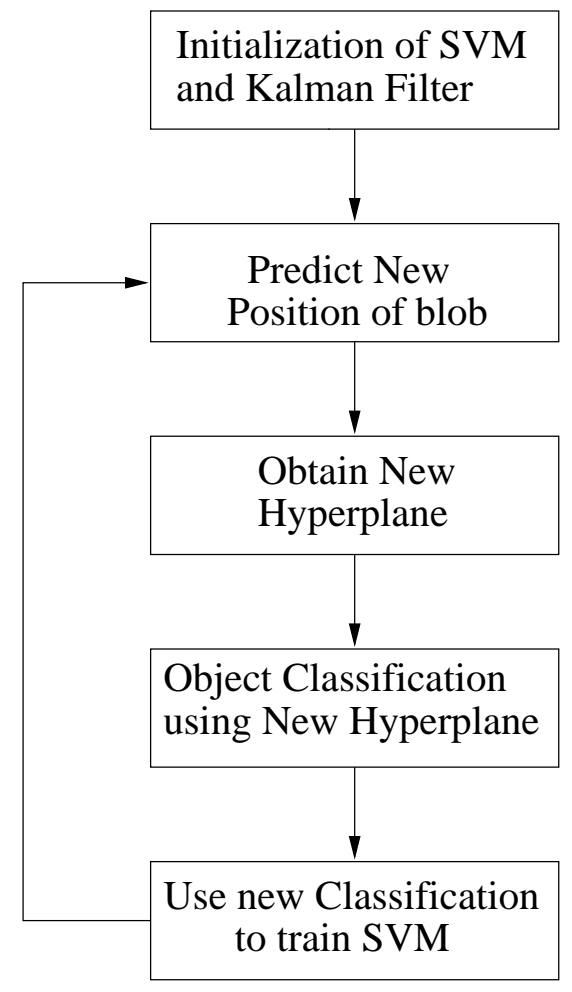

Figure 5: Block diagram Showing the Adaptive Learning Algorithm

\subsection{Step 5: Obtain the Filtered Support Vectors}

To capture the changes in the lighting condition and the unexpected changes in the position of the blob, a new set of support vectors is obtained. That is we feed the classified data to our training algorithm and filter any errors that might have been there in our prediction algorithm. However to obtain the new set of support vectors, we need only consider those points which lie close to the hyperplane. Thus saving computation without compromising the robustness.

This step is extremely important because it ensures handling any gradual change in the lighting condition and any error in the motion estimation. e.g As the hand moves, because of the different lighting conditions, the skin may experience slight changes in color. Thus, this is taken care of by retraining the SVMs.

We also need to update the Kalman filter equations. i.e. solve

$$
\begin{gathered}
K_{k}=P_{k}^{-}\left(P_{k}^{-}+R_{k}\right)^{-1} \\
P_{k}=\left(I-K_{k}\right) P_{k}^{-}
\end{gathered}
$$

To update the Kalman filter gain and the error covariance matrix. We also need to update the states using the measurement.

$$
\hat{\mathbf{p}}_{k}=\hat{\mathbf{p}}_{k}^{-}+K\left(z_{k}-\hat{\mathbf{p}}_{k}^{-}\right)
$$

Once the support vectors are obtained we go back to step 2. This algorithm is demonstrated in the flowchart given in Fig 9.

\section{Results}

The results obtained from the experiments demonstrate the abilities of this tracking approach. We present three experiments, first two of which are on synthetically generated data using real images and the third is on a real video sequence. These experiments were done using linear kernel function for SVM which handles the translation of objects on the image plane.

\subsection{Experiments on Synthetic Data}

In this experiment both motion and changes in color were applied to the objects. When motion is applied to the object, we expect the location of the positive (object's) support vectors to remain in the same physical location on the object. We also tested the algorithm on the images, knowing the real motion beforehand. The SVM of the original image was updated using the motion. When small variation of color are applied, we expect the SV's to remain in the same location. In the experiments, subsampled images of size 90x90 were used. The training of the SVM was done using the software $S V M^{\text {light }}$ [4]. The kernel funtion we used was the simple dot product, which was sufficient in these cases.

Fig 6 and 7 show the results for the hand and face tracking respectively. Fig 6(a), 7(a) show the original 


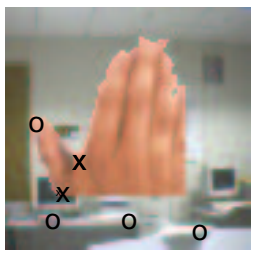

(a)

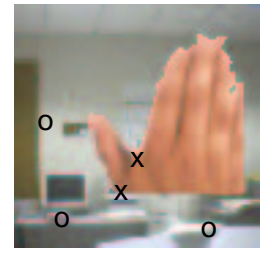

(b1)

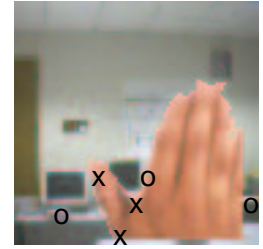

(c1)

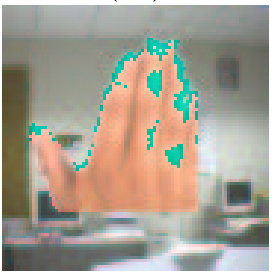

(d1)

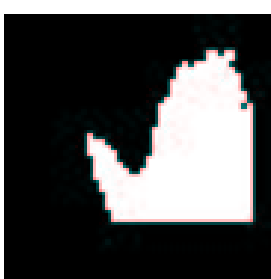

(b2)

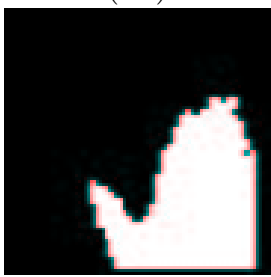

(c2)

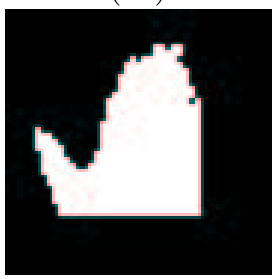

(d2)

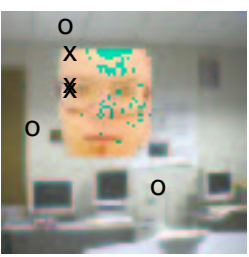

(a)

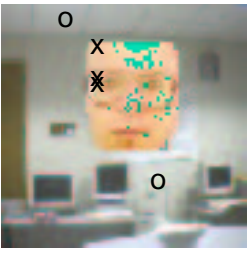

(b1)

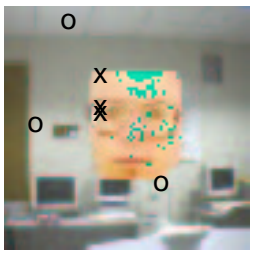

(c1)

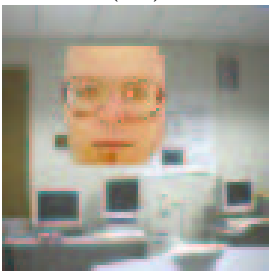

(d1)

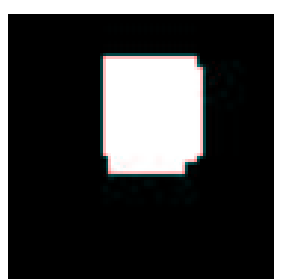

(b2)

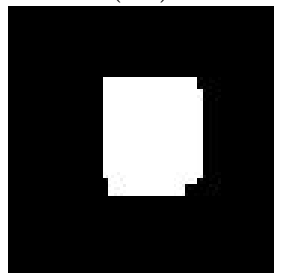

(c2)

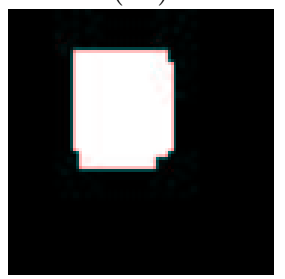

(d2)
Figure 6: Fig(a) shows the hand which is the object to be tracked. ' $x$ ' are the support vectors belonging to hand after training. 'o' represents the -ve support vectors. b1,b2 shows the hand, and the classified output, as it has moved to right by 18 pixels, c1,c2 shows the hand and the output after the lateral movement by 18 pixels in both $x$ and $y$. d1,d2 shows the tracking results as the color of the hand changes.

hand and face positions and the locations of the support vectors, Fig 6(b1),7(b1) show the image after a small (18 and 10 horizontal pixel) change in the hand and face position, and the locations of the SV learned in this location.Fig $6(\mathrm{c} 1), 7(\mathrm{c} 1)$ show the image after a bigger motion (18, 10 horizontal and 18, 10 vertical pixels) and the SV learned in this location. It can be seen that, as expected, the positive SV maintained their location on the object, and the negative SV's did
Figure 7: Fig(a) shows the face which is the object to be tracked. ' $x$ ' are the support vectors belonging to the face after training. 'o' represents the -ve support vectors. b1,b2 shows the face, and the classified output, as it has moved to right by 10 pixels, c1,c2 shows the face and the output after the lateral movement by 10 pixels in both $x$ and $y$. d1, d2 shows the tracking results as the color of the face changes.

not change as well, as long as the object did not obscure them after the motion. Now, using the Kalman filter, the original SVs were updated and after obtaining the new hyperplane, classification was done. Fig $6(\mathrm{~b} 2), 7(\mathrm{~b} 2)$ and $6(\mathrm{c} 2), 7(\mathrm{c} 2)$ show the classification of the object pixels (white) vs. the background pixels (black) after the movement. Not even a single pixel was misclassified in all of these cases. Fig 6(d1), 7(d1) demonstrate the variation of color on the classification. 


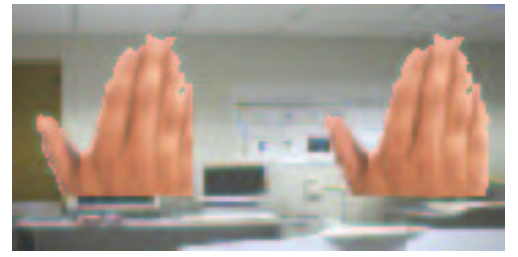

(a)

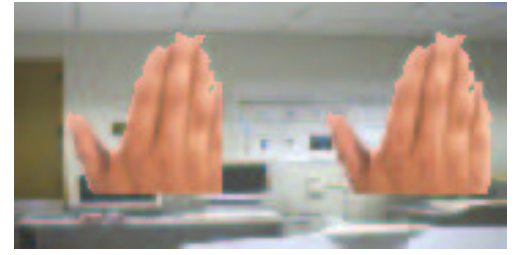

(b)

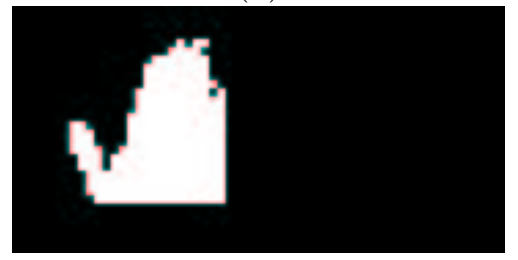

(c)

Figure 8: a) shows the image with two identical hands of which left is to be tracked. b) shows the image after the left hand has moved by 10 pixels in $\mathrm{x}$ direction. $\mathrm{c}$ ) is the output after classification.

In both cases the color of the object was changed by $10 \%$ in all of the color components. Classification of the hand and face were performed using the SVM before the color change, and there were no misclassified pixels. This can be seen in Fig 6(d2), 7(d2).

The next experiment involved classification and tracking of an object in the presence of a similar object in the background. Using only a color based classifier is assured to fail in this case. Fig 8(a) shows the image of two identical hands placed in a different location in the image, and the left hand is the one we want to track. Using both location and color, a SVM is trained. In Fig 8(b) a movement is applied to the left hand ( 10 pixels horizontaly), and the SVM is updated accordingly using the Kalman filter. Classification of the image is then performed using the updated SVM, and the result is shown is Fig 8(c). Only 2 pixels were misclassified as background (out of 1800 total pixels in the image )

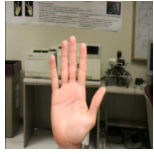

(a1)

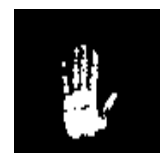

(b1)

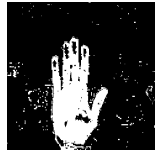

(c1)

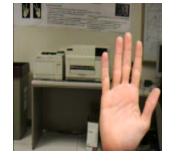

(a2)

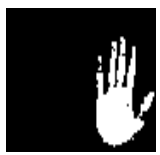

(b2)

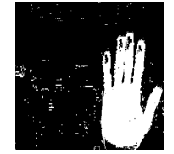

$(\mathrm{c} 2)$

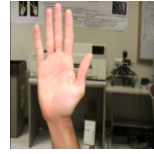

(a3)

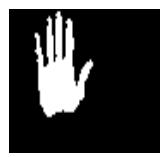

(b3)

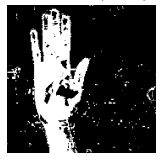

(c3)
Figure 9: Fig (a1),(a2),(a3) shows three frames from the video at different times. Fig (b1),(b2),b(3) shows the segmentation obtained by using SVM based tracker for these frames. Fig (c1),(c2),(c3) shows the segmentation obtained by using single Gaussian color based tracker.

\subsection{Experiments on Real Video}

The final set of experiments are on the real video sequence of hand motion. The sequence is of length 250 frames and the result of tracking on some of these is presented along with the comparison to color based blob tracking. The updating of SVM and the selection of feature vectors is done in the way as mentioned in the previous section. For the color based blob tracking we have used single Gaussian normalized RGB [13] model. For the purpose of learning the initial SVM we used background subtraction technique. The results 9 show that SVM based tracking (segmentation of the object from the background) outperforms the latter.

It should be noted that the run time of the classification was in the order miliseconds, and the training of the SVM was in the order of parts of a second ( 0.1 second). Keeping in mind that this was not attempted to be made real time as of yet, these initial run times show the feasibility of a real time tracking system.

\section{Summary}

In this paper we introduced a new approach in feature tracking. SVM has been shown to be a strong tool for binary classification. The adaptive learning approach of SVM can be very helpful for classification of objects which are expected to change over time. We have 
shown in the experiments that this adaptive learning can be implementated for feature tracking, and thus exploiting the benefits of the SVM classifiers. The results show taht the translation motion of the objects can be handled well by using linear kernel function for SVM. Future work will explore using different kernel functions to address the problem of rotation, scaling and small shape changes. By keeping track of the movement of the hyperplane, we can develop a framework where this data is used to recognize the gestures. Infact this can also be used in multimodal framework where we want to use different modalities. The vector $\mathbf{x}$ can have fields corresponding to different modalities and the output of all those modalities is used to control the system.

\section{Acknowledgements}

This work was supported in part by National Science Foundation Grants CDA-96-24396 and IRI-96-34618. The authors will also like to thank the reviewers for their useful comments.

\section{References}

[1] Azoz Y., Devi L., Sharma R., "Reliable Tracking of Human Arm Dynamics by Multiple Cue Integration and Constraint Fusion", CVPR'98, June 1998.

[2] Boser B., Guyon I., Vapnik V., "A Training Algorithm for Optimal Margin Classifiers",Fifth Annual Workshop on Computational Learning Theory. ACM Press, Pittsburgh, 1992.

[3] Burges C., "A Tutorial on Support Vector Machines for Pattern Recognition", Data Mining and Knowledge Discovery, 1998.

[4] Joachims T.,"Making large-Scle SVM learninf Practical", Advance in Kernel Methods- Support Vector Learning, MIT-Press, 1999.

[5] Osuna E., Freund R., Girosi F., "Training Support Vector Machine: an Application to Face Detection", CVPR'9\%, June 17-19, 1997.

[6] Osuna E., Freund R., Girosi F., "An Improved Training Algorithm for Support Vector Machines", Proc. of IEEE NNSP'97, Sep. 1997.

[7] Pavlovic V., Sharma R., Huang T. S., "Visual interpretation of hand gestures for human- computer interaction: A review", IEEE Trans. on PAMI, July 1997.

[8] Sharma R., Pavlovic V. I., and Huang T. S., "Toward Multimodal Human-Computer Interface", Proc. IEEE special issue on Multimedia Computing and Communication, Aug. 1997.

[9] Smola A., Scholkopf B, "A Tutorial on Support Vector Regression", NeuroCOLT2 Technical Report Series, October'1998.

[10] Vapnik V., Lerner A., "Pattern Recognition using Generalized Portrait Method", Automation and Remote Control,24,1963.

[11] Vapnik V., "The Nature of Statistical Learning Theory", Springer-Verlag, New York, 1995.

[12] Anderson B. D. O. and Moore J. B., "Optimal Filtering", Prentice Hall, New Jersey, 1979.

[13] Yang J. and Waibel A., "A real-time face tracker",Proc. of 3rd Workshop on Appl. of Comp. Vision, Sarasota, FL, 1996.

[14] Christopher R. Wren, Ali Azarbayejani, Trevor Darrell, Alex Pentland " Pfinder: Real-Time Tracking of the Human Body", IEEE Transactions on Pattern Analysis and Machine Intelligence, July 1997. 